\title{
Efficient mutagenesis by CRISPR/Cas system during meiotic maturation of porcine oocytes
}

\author{
Asuka ONUMA ${ }^{1)}$, Wataru FUJII'), Koji SUGIURA ${ }^{1)}$ and Kunihiko NAITO ${ }^{1)}$ \\ 1) Department of Animal Resource Sciences, Graduate School of Agricultural and Life Sciences, The University of Tokyo, \\ Tokyo 113-8657, Japan
}

\begin{abstract}
Genome editing using the CRISPR/Cas system can induce mutations with high efficiency, and allows easier production of genome-modified animals than that offered by the conventional method where embryonic stem cells are used. However, studies using CRISPR/Cas systems have been mostly limited to proliferating somatic cells and pronuclear-stage fertilized eggs. In contrast, the efficiency of a CRISPR/Cas system in immature and maturing oocytes progressing through meiosis has not yet been assessed. In the present study, we evaluated the genome-modification efficiency of the CRISPR/Cas system during meiotic maturation of porcine oocytes. Additionally, the localization of the Cas9 protein in immature oocytes was analyzed in relation to nuclear transport and mutation induction. The results showed that CRISPR/Cas induced mutation with high efficiency even in maturing oocytes with condensed chromosomes, whereas mutations were not induced in GVstage oocytes. The localization analysis of enhanced green fluorescent protein (EGFP)-tagged Cas9 (Cas9-EGFP) revealed that the nuclei contained lesser Cas 9 than the cytoplasm in immature oocytes. Treatment with leptomycin B, a nuclear export inhibitor, increased the amount of nuclear Cas 9 and enabled mutation induction in GV oocytes. Our results suggest that CRISPR/Cas systems can be applied to oocytes during meiotic maturation and be implemented in novel applications targeting female genomes.
\end{abstract}

Key words: CRISPR/Cas9, Germinal vesicle, Nuclear transport, Porcine oocyte

(J. Reprod. Dev. 63: 45-50, 2017)

$\mathbf{R}$ ecent years have witnessed the prominent development of genomeengineering techniques using artificial nucleases; these techniques allow easier, faster, and cheaper production of genome-modified animals compared to the one achieved through the conventional method, i.e., based on homologous recombination in embryonic stem cells [1]. Especially, genome editing using bacterial CRISPR/ Cas systems is highly efficient in creating mutations and has been extensively used in a large number of studies [2, 3]. A CRISPR/Cas system consists of a guide RNA (gRNA) that recognizes a target DNA sequence, and the Cas 9 endonuclease; the latter is recruited by the gRNA to create a double-strand break at the target DNA sequence, which is repaired by the error-prone non-homologous end-joining (NHEJ) reaction, leading to mutation in the target sequence.

At present, many genome-modified animals, including classic experimental animals such as mice and rats, as well as livestock animals such as pigs and sheep, have been produced by CRISPR/ Cas systems. In all cases CRISPR/Cas were introduced into fertilized eggs at the pronuclear stage [4-10], with the exception of a single study, in which Cas 9 mRNA and gRNA were introduced into mouse mature oocytes just before fertilization [11]. Genome modification

Received: June 23, 2016

Accepted: September 30, 2016

Published online in J-STAGE: October 21, 2016

C2017 by the Society for Reproduction and Development

Correspondence: K Naito (e-mail: aknaito@mail.ecc.u-tokyo.ac.jp) and

W Fujii (e-mail: awtrfj@mail.ecc.u-tokyo.ac.jp)

This is an open-access article distributed under the terms of the Creative Commons Attribution Non-Commercial No Derivatives (by-nc-nd) License

$<$ http://creativecommons.org/licenses/by-nc-nd/4.0/>. by CRISPR/Cas systems has also been studied in proliferating cell cultures of various species; however, these techniques have never been studied in immature and maturing oocytes progressing through meiosis.

The high mutation-inducing efficiency of CRISPR/Cas systems has been substantially validated in developing embryos and proliferating somatic cells, which are going through repeated cycles composed of a long interphase, during which the chromosomes are decondensed, and a short M-phase characterized by the presence of condensed chromosomes [2, 3]. Cas9 proteins and gRNA are generally considered to enter the nucleus together and attack the decondensed interphase chromosomes, although the identity of the exact cell phases in which CRISPR/Cas systems function has never been examined systematically. Therefore, it has remained unclear whether CRISPR/Cas systems work efficiently in the large nuclei of immature oocytes arrested at meiotic prophase, or on the condensed chromosomes of maturing oocytes progressing through meiosis.

In the present study, we validated the genome-modification efficiency of a CRISPR/Cas system during meiotic maturation of porcine oocytes and described the characteristics of the induced mutations. We also analyzed the localization of Cas 9 protein in immature oocytes in relation to nuclear transport capacity and mutation induction capacity.

\section{Materials and Methods}

\section{Construction of the gRNA coding vector}

As shown in Fig. 1A, the gRNA was designed to target exon 8 of the porcine GGTA1 gene, which encodes $\alpha 1$,3-galactosyltransferase; 
A

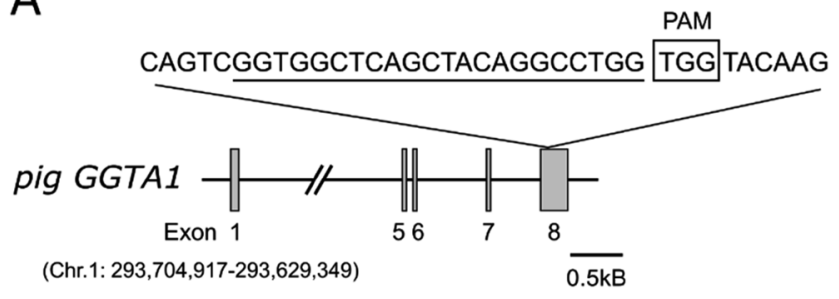

B

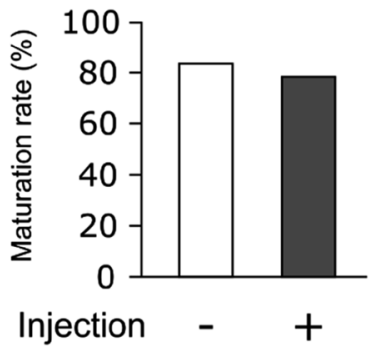

Fig. 1. Construction of the used CRISPR/Cas system and the effects of guide RNA (gRNA) and Cas 9 mRNA introduction into porcine oocytes. (A) Guide RNA was designed to target exon 8 of the GGTA1 gene. The underlined nucleotides and the boxed sequences indicate the gRNA binding site and the PAM domain, respectively. $(\mathrm{B}, \mathrm{C})$ Porcine immature oocytes were injected into cytoplasm with gRNA and Cas 9 mRNA shortly after collection from ovarian follicles and cultured for $44 \mathrm{~h}$. Maturation rates were evaluated as the percentages of oocytes that had reached the second meiotic metaphase by the end of the 44-hour incubation (B). The 44-h cultured oocytes were parthenogenetically activated by electrostimulation, then cultured for an additional 2 days. Cleavage rates were evaluated as the percentages of embryos that had developed to the two-cell stage or further by the end of the 2-day period (C). The numbers of examined oocytes and embryos were higher than 80 .

a plasmid vector coding the GGTA1-gRNA with T3 promoter was synthesized as described in a previous study [6]. This vector was sequenced using a commercial sequencing kit (Applied Biosystems, Foster City, CA, USA) and a DNA sequencer (Applied Biosystems) according to the manufacturer's instructions.

\section{In vitro transcription of Cas $9 \mathrm{mRNA}$ and $g R N A$}

For in vitro synthesis of $\operatorname{Cas} 9 \mathrm{mRNA}$ and enhanced green fluorescent protein (EGFP)-tagged Cas9 (Cas9-EGFP) mRNA, the coding vectors reported in a previous study [6] were linearized by $S p h \mathrm{I}$ and transcribed in vitro by T3 RNA polymerase (Promega, Fitchburg, WI, USA) in the presence of $\mathrm{m} 7 \mathrm{G}\left(5^{\prime}\right) \mathrm{ppp}\left(5^{\prime}\right) \mathrm{G}$, so that the synthesized RNA transcripts would be capped. In the case of gRNA, its coding vector was linearized by DraI and transcribed by the same procedure, but without $\mathrm{m} 7 \mathrm{G}\left(5^{\prime}\right) \mathrm{ppp}\left(5^{\prime}\right) \mathrm{G}$ to avoid the formation of capped transcripts. EGFP mRNA was prepared as described previously [12]. The RNA transcripts were precipitated with absolute ethanol, washed, and resuspended in RNase-free water. The RNA solutions were stored at $-80^{\circ} \mathrm{C}$ until use.
Collection and in vitro maturation of porcine oocytes

Ovaries were collected from prepubertal gilts at a commercial slaughterhouse and transported to our laboratory in saline. Cumulusoocyte complexes (COCs) were aspirated from follicles, and washed with the "maturation medium", which was modified North Carolina State University Medium 37 (mNCSU37) containing 10\% porcine follicular fluid. COCs were then cultured in the maturation medium supplemented with $10 \mathrm{IU} / \mathrm{ml}$ equine chorionic gonadotropin (eCG; ASKA Pharmaceutical, Tokyo, Japan), $10 \mathrm{IU} / \mathrm{ml}$ human chorionic gonadotropin (hCG; ASKA Pharmaceutical), and $1 \mathrm{mM}$ dibutyryl cyclic adenosine 3',5'-monophosphate (dbcAMP; Sigma-Aldrich, St. Louis, MO, USA) for $20 \mathrm{~h}$, followed by an additional $24 \mathrm{~h}$ incubation in the maturation medium without hormones or dbcAMP. To produce GV-arrested oocytes, cells were cultured for $44 \mathrm{~h}$ in the maturation medium containing $100 \mu \mathrm{M}$ roscovitine (Cell Signaling Technology, Danvers, MA, USA), an inhibitor of CDK1 activity, instead of dbcAMP. In the experiments where nuclear export was inhibited, $200 \mathrm{nM}$ of the exportin-1 inhibitor, leptomycin B (LMB; Merck Millipore, Darmstadt, Germany), were added to the maturation medium. All cultures were incubated at $37{ }^{\circ} \mathrm{C}$, in a saturated (100\%) humidity, $5 \% \mathrm{CO}_{2} / 95 \%$ air atmosphere. After incubation, the surrounding cumulus cells were removed by repeated pipetting. Some denuded oocytes were examined for nuclear status after being fixed with $25 \%$ acetic acid in ethanol and stained with a $0.75 \%$ aceto-orcein solution.

\section{Parthenogenetic activation and in vitro culture of oocytes}

After in vitro maturation, the denuded oocytes were placed in a solution containing $280 \mathrm{mM}$ mannitol, $0.05 \mathrm{mM} \mathrm{CaCl}_{2}, 0.1 \mathrm{mM}$ $\mathrm{MgSO}_{4}$, and $0.01 \% \mathrm{BSA}$, and then activated by a single DC pulse of $150 \mathrm{~V} / \mathrm{mm}$ for $100 \mu \mathrm{sec}$. The oocytes were cultured in mNCSU37 containing $0.4 \%$ BSA and $2.2 \mu \mathrm{g} / \mathrm{ml}$ cytochalasin B (Sigma-Aldrich) for $3 \mathrm{~h}$ in order to inhibit the formation of the second polar body, then incubated for another $48 \mathrm{~h}$ in cytochalasin B-free medium. Cleavage rates were calculated from the percentages of zygotes that had reached the 2-cell or a later stage at $48 \mathrm{~h}$ after activation.

\section{Microinjection}

Microinjection was performed using an inverted microscope equipped with a microinjector (Narishige, Tokyo, Japan). Approximately $50 \mathrm{pl}$ of an RNA solution containing $20 \mathrm{ng} / \mu \mathrm{l}$ gRNA, $50 \mathrm{ng} / \mu \mathrm{l}$ Cas 9 mRNA, and $50 \mathrm{ng} / \mu \mathrm{l} E G F P$ mRNA were injected into each ooplasm of the non-cultured porcine COCs using continuous pneumatic pressure. After injection, COCs were cultured for $44 \mathrm{~h}$ as described above. Only oocytes expressing EGFP were collected and subjected to sequencing analysis. In the experiments where Cas 9 localization was analyzed, $20 \mathrm{ng} / \mu \mathrm{gRNA}$ and $400 \mathrm{ng} /$ $\mu 1$ Cas $9-E G F P$ mRNA were injected using the same procedure.

\section{Analysis of induced mutations in target sequence}

Each collected oocyte was treated with proteinase $\mathrm{K}$ (Roche, Basel, Switzerland), then put into a single PCR tube and subjected to PCR using the following primers: Forward: 5'- AAGAGTGAGAGAGGAAGGAGCAG-3'; Reverse: 5'ACATGCCTATATGATAATCCCAGCA-3'. PCR products were purified by agarose gel electrophoresis. The extracted fragments were 
directly sequenced as described above. The predicted mutation pattern for each allele of each single oocyte was derived from sequence data according to a previously described protocol [6].

\section{Localization analysis of Cas9}

The injected COCs were cultured for $44 \mathrm{~h}$ in the presence of $100 \mu \mathrm{M}$ roscovitine to arrest the cells at the GV stage. After Cas9 expression, the injected oocytes were fixed in $3.5 \%$ paraformaldehyde for $1 \mathrm{~h}$, permeabilized in $0.1 \%$ Triton $\mathrm{X}-100$ for $20 \mathrm{~min}$, and stained with $100 \mu \mathrm{g} / \mathrm{mL}$ propidium iodide for $30 \mathrm{~min}$. All reagents were dissolved in phosphate-buffered saline (PBS) containing $0.1 \%$ polyvinylpyrrolidone (PVP). The oocytes were mounted on a glass slide and then examined under a confocal laser scanning microscope (LSM 700; Carl Zeiss Microscopy, Jena, Germany). The examined oocytes were given a score based on the ratio of fluorescence intensity between the nucleus and the cytoplasm.

\section{Statistical analysis}

The results regarding maturation and cleavage rates were evaluated with the chi-square test, whereas Student's $t$-test was used for localization scores. P-values lower than 0.05 were considered statistically significant.

\section{Results}

\section{Effects of CRISPR/Cas introduction into porcine immature} oocytes on meiotic maturation and subsequent early cleavage

We first examined the effects of introducing CRISPR/Cas into porcine immature oocytes on maturation and cleavage rates. The maturation rate of the oocytes that had been microinjected with gRNA and $\operatorname{Cas} 9$ mRNA just after collection from ovarian follicles and then cultured for $44 \mathrm{~h}$ was not significantly different from that of non-injected oocytes (78.0\% vs. $83.5 \%$; Fig. 1B). The cleavage rate of the injected oocytes 2 days after parthenogenetic activation by electrical stimulation was also comparable to that of the non-injected experimental group (42.2\% vs. 50.0\%; Fig. 1C). The results indicate that the injection of these RNAs into porcine immature oocytes has few or no deleterious effects, at least with respect to meiotic maturation and the subsequent early cleavage.

\section{Efficiency of mutation induction by the CRISPR/Cas system in porcine maturing oocytes}

The mutation-inducing efficiency was evaluated $44 \mathrm{~h}$ after the injection of the CRISPR/Cas system into porcine immature oocytes. About $81.0 \%$ of injected oocytes exhibited mutations at the target loci (Table 1), indicating that the mutation-inducing efficiency of the CRISPR/Cas system during the meiotic maturation of the oocytes was high. Mutations were detected in only one or two alleles in $61.8 \%$ and $35.5 \%$ of the oocytes, respectively (Table 1); the mutation lengths were smaller than 10 bases in most cases, while the maximum observed length was 44 bases (Table 2). These results suggest that when introduced during meiotic maturation, the CRISPR/Cas system is predisposed to induce short and preferentially heterozygous mutations.

To determine whether the mutations were induced before or after chromosome condensation, the oocytes were treated with roscovitine, an inhibitor of CDK1 activity, to inhibit germinal vesicle breakdown (GVBD), blocking chromosome condensation. The mutation rate of $\mathrm{GV}$-arrested oocytes was examined $44 \mathrm{~h}$ after injection of the CRISPR/ Cas system; results revealed that no mutations had been induced in the oocytes (Table 1), indicating that the mutations observed in the previous experiments, i.e., in mature oocytes, were induced after the GVBD and chromosome condensation had taken place.

\section{Effects of nuclear-export inhibition on Cas9 localization and mutation-inducing efficiency}

Next, we examined whether the absence of mutations in the $\mathrm{GV}$-arrested oocytes resulted from the unusual chromosome configuration at the GV stage, or from differences in the expression or localization of the Cas9 protein. Cas9-EGFP mRNA was injected into GV-arrested oocytes, and cells were classified into three levels based on the ratio of EGFP signal strength between the nucleus $(\mathrm{GV})$ and the cytoplasm, as shown in Fig. 2A. In almost all oocytes, EGFP fluorescence was lower in the nuclei than in the cytoplasm (Fig. 2B, score 1 and 2), indicating that the absence of mutations in $\mathrm{GV}$-arrested oocytes may be the result of insufficient nuclear Cas9. To test this hypothesis, we used LMB, which is a specific inhibitor of the nuclear-export receptor, exportin-1. As expected, the inhibition of nuclear export by LMB caused a significant increase in the nuclear levels of Cas9, as demonstrated by the increase in EGFP nuclear fluorescence (Fig. 2B, 2C).

Mutation induction was examined in the GV-arrested / LMB-treated oocytes, revealing that $16.7 \%$ of these oocytes were mutated at the target loci (Table 3), in contrast to the absence of mutations in the GV-arrested oocytes that had not been treated with LMB. This shows that the increase in nuclear Cas9 enables mutation induction in GV oocytes. The mutations were found in only one allele in four of the five oocytes, and two alleles in the remaining oocyte (Table 3 ), confirming the disposition for inducing heterozygous mutations during meiotic maturation. The mutation lengths were smaller than 10 bases in 4 cases, but a 170-base mutation was detected in two cases (Table 4).

\section{Discussion}

In the present study, the possibility of using a CRISPR/Cas system to induce mutations during meiotic maturation was examined using in vitro maturation culture of porcine oocytes. The results suggested that highly efficient mutation induction by CRISPR/Cas is possible even in maturing oocytes with condensed chromosomes. We also demonstrated that GV-stage oocytes are unfavorable targets for mutation induction, while increasing the amount of nuclear Cas9 through nuclear export inhibition enables mutation induction in GV oocytes. The injection of RNAs into porcine immature oocytes in itself had no or few deleterious effects, at least with respect to the meiotic maturation rate and the subsequent early cleavage rate. In the studies published to date, the mutation efficiency of CRISPR/ Cas systems depended on the target sequences, but often almost all embryos or offspring were mutated [5-8, 13]. Our data demonstrated that the efficiency of mutation induction in meiotic maturing oocytes by the used CRISPR/Cas system was $80 \%$ or higher, which is comparable to previously reported rates. Our results indicate that 
Table 1. Mutation rates and numbers of mutated alleles in CRISPR/Cas treated porcine oocytes

\begin{tabular}{ccccccc}
\hline \multirow{2}{*}{$\begin{array}{c}\text { Stage of analyzed } \\
\text { oocyte }\end{array}$} & Analyzed oocyte & $\begin{array}{c}\text { Mutated oocyte } \\
\text { (\%) }\end{array}$ & \multicolumn{4}{c}{$\begin{array}{c}\text { Oocyte with indicated number of mutated alleles } \\
\text { (\% of mutated oocyte) }\end{array}$} \\
\cline { 3 - 7 } & & & 1 & 2 & 3 & 4 \\
\hline MII & 42 & 34 & 21 & 12 & 0 & 1 \\
& 15 & $(81.0)$ & $(61.8)$ & $(35.3)$ & $(0)$ & $(2.9)$ \\
\hline GV & 15 & 0 & 0 & 0 & 0 \\
& & $(0)$ & $(0)$ & $(0)$ & $(0)$ & $(0)$ \\
\hline
\end{tabular}

Table 2. Sequences of mutated alleles in porcine MII oocytes

\begin{tabular}{|c|c|c|}
\hline Sequence of target site* & Indel (base) & Number of alleles \\
\hline CAGTCGGTGGCTCAGCTACAGGCCTGGTGGTACAAGGCACATCCTGACGA & WT & \\
\hline CAGTCGGTGGCTCAGCTACAGGC-TGGTGGTACAAGGCACATCCTGACGA & -1 & 7 \\
\hline CAGTCGGTGGCTCAGCTACAGGCC-GGTGGTACAAGGCACATCCTGACGA & -1 & 1 \\
\hline CAGTCGGTGGCTCAGCTACAGGtCTGGTGGTACAAGGCACATCCTGACGA & $-1,+1$ & 1 \\
\hline CAGTCGGTGGCTCAGCTACAGG--TGGTGGTACAAGGCACATCCTGACGA & -2 & 3 \\
\hline CAGTCGGTGGCTCAGCTACAGGCtgaTGGTGGTACAAGGCACATCCTGACGA & $-1,+3$ & 1 \\
\hline CAGTCGGTGGCTCAGCTACAGGCtgaGGTGGTACAAGGCACATCCTGACGA & $-2,+3$ & 1 \\
\hline CAGTCGGTGGCTCAGCTACAGGCtgaGTGGTACAAGGCACATCCTGACGA & $-3,+3$ & 1 \\
\hline CAGTCGGTGGCTCAGCTACAGGC-tgaTGGTACAAGGCACATCCTGACGA & $-4,+3$ & 1 \\
\hline CAGTCGGTGGCTCAGCTACAGG-----TGGTACAAGGCACATCCTGACGA & -5 & 6 \\
\hline CAGTCGGTGGCTCAGCTAC-----TGGTGGTACAAGGCACATCCTGACGA & -5 & 1 \\
\hline CAGTCGGTGGCTCAGCTACAGGCacatccTGGTGGTACAAGGCACATCCTGA & $-1,+6$ & 1 \\
\hline CAGTCGGTGGCTCAGCTACAGGCCaccgacTGGTACAAGGCACATCCTGACG & $-3,+6$ & 1 \\
\hline CAGTCGGTGGCTCAGCTACAGGCCaccgtagTGGTGGTACAAGGCACATCCT & +7 & 1 \\
\hline CAGTCGGTGGCTCAGCTACAGGCCTGtactgggGTGGTACAAGGCACATCCT & +7 & 1 \\
\hline CAGTCGGTGGCTCAGCTACAGG--------TACAAGGCACATCCTGACGA & -8 & 1 \\
\hline CAGTCGGTGGCTCAGCT--------GGTGGTACAAGGCACATCCTGACGA & -8 & 1 \\
\hline CAGTCGGTGGCTCAGCTA-------tgTGGTACAAGGCACATCCTGACGA & $-9,+2$ & 1 \\
\hline CAGTCGGTGGCTCAG-----gatgTGGTGGTACAAGGCACATCCTGACGA & $-9,+4$ & 1 \\
\hline CAGTCGGTGGCTCAGCTA----tcgatTGGTACAAGGCACATCCTGACGA & $-9,+5$ & 1 \\
\hline CAGT-------ggAGCTACAGGCCaccgacTGGTGGTACAAGGCACATCCTG & $(-9,+2),+6$ & 1 \\
\hline -----GGTGGCTCAGCTACAGGCacatcctgTGGTGGTACAAGGCACATCCT & $-10,(-1,+8)$ & 1 \\
\hline CAGTCGGTGGCTC------------GGTGGTACAAGGCACATCCTGACGA & -12 & 1 \\
\hline CAGTCGGTGGCTCAGCTACAGGC--------------ACATCCTGACGA & -15 & 2 \\
\hline CAGTCGGTGG-----------------TGGTACAAGGCACATCCTGACGA & -17 & 2 \\
\hline CAGTCGGTGG--------------------TACAAGGCACATCCTGACGA & -20 & 6 \\
\hline 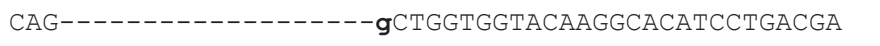 & $-20,+1$ & 1 \\
\hline 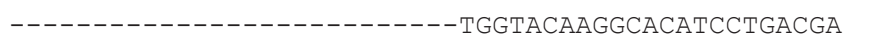 & -43 & 1 \\
\hline---------------------------- ACAAGGCACATCCTGACGA & -44 & 1 \\
\hline CAGTCGGTGGCTCAGCTACAGGCgaccaacaaggtacagggggta & undefined & 1 \\
\hline
\end{tabular}

* The underlined nucleotides in the wild-type (WT) sequence shows a PAM domain. Small letters and hyphens indicate inserted and deleted nucleotides compared to WT, respectively.

maturing oocytes can be efficiently subjected to genome modification though CRISPR/Cas systems, although further studies with different target sequences are necessary for confirmation.

In the previous studies, CRISPR/Cas systems were applied to proliferating cells or developing embryos, which are cells progressing through repeated cycles of long interphases and short M-phases $[2,3]$. Although the introduction of gRNA and $\operatorname{Cas} 9$ mRNA into mature oocytes suggested that CRISPR/Cas could also work before or at the pronuclear stage [11], the applicability of CRISPR/Cas to condensed chromosomes during M-phase had not been clearly shown. In the present study, the CRISPR/Cas system was unable to induce mutations in GV-arrested oocytes unless nuclear export had been inhibited, indicating that the mutations found in mature oocytes were induced after GVBD. Our results are the first to suggest that a CRISPR/Cas system can induce mutations in condensed chromosomes during M-phase. 
A

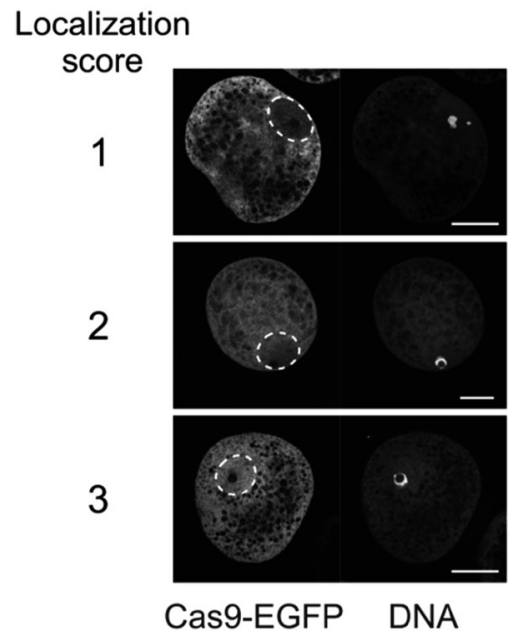

B

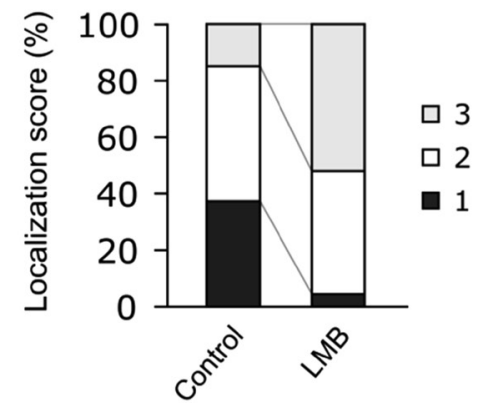

C

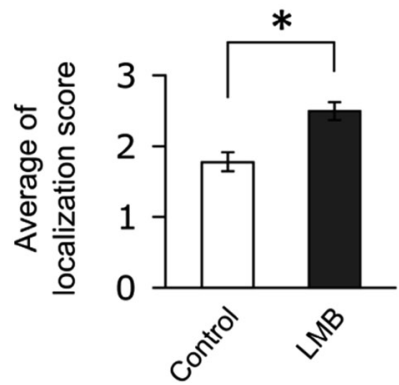

Fig. 2. Effects of nuclear export inhibition on Cas9 localization. Cas9-EGFP mRNA was injected into porcine immature oocytes which were then cultured for $44 \mathrm{~h}$ with roscovitine in order to arrest at the GV stage. Cas 9 localization was evaluated by EGFP fluorescence. (A) The ratio of EGFP signal strength between GV and cytoplasm was classified into three levels as localization scores. Typical examples are shown, accompanied by images of the same cells after propidium iodide DNA staining. Dashed lines show GV outlines. Scale bars indicate $50 \mu \mathrm{m}$. (B) Ratios of localization scores of oocytes cultured with or without LMB, an inhibitor of exportin-1, are shown. More than 25 oocytes were examined in each experimental group. (C) Average (mean $\pm \mathrm{SEM}$ ) values of the localization scores shown in $\mathrm{B} ; * \mathrm{P}<0.05$.

Table 3. Mutation rates and number of mutated alleles in porcine oocytes cultured with leptomycin B

\begin{tabular}{ccccccc}
\hline \multirow{2}{*}{$\begin{array}{c}\text { Stage of analyzed } \begin{array}{c}\text { Mocyte } \\
\text { oocyted oocyte }\end{array} \\
\text { MII }\end{array}$} & Analyzed oocyte & $\begin{array}{c}\text { Mutate with indicated number of mutated alleles } \\
\text { (\% of mutated oocyte) }\end{array}$ \\
\cline { 3 - 6 } & 15 & 12 & 1 & 2 & 3 & 4 \\
\hline GV (+ LMB) & 30 & $(80.0)$ & $(75.0)$ & $(25.0)$ & $(0)$ & $(0)$ \\
& & 5 & 4 & 1 & 0 & 0 \\
& & $(16.7)$ & $(80.0)$ & $(20.0)$ & $(0)$ & $(0)$ \\
\hline
\end{tabular}

Table 4. Sequence of mutated alleles in porcine MII oocytes and leptomycin B-treated GV oocytes

\begin{tabular}{|c|c|c|c|}
\hline $\begin{array}{l}\text { Stage of analyzed } \\
\text { oocytes }\end{array}$ & Sequence of target site* & Indel (base) & Number of alleles \\
\hline \multirow{12}{*}{ MII } & CAGTCGGTGGCTCAGCTACAGGCCTGGTGGTACAAGGCACATCCTGACGA & WT & \\
\hline & CAGTCGGTGGCTCAGCTACAGGC-TGGTGGTACAAGGCACATCCTGACGA & -1 & 2 \\
\hline & CAGTCGGTGGCTCAGCTACAG-CCTGGTGGTACAAGGCACATCCTGACGA & -1 & 1 \\
\hline & CAGTCGGTGGCTCAGCTACAGGC--GGTGGTACAAGGCACATCCTGACGA & -2 & 1 \\
\hline & CAGTCGGTGGCTCAGCTACAGGCtgagTGGTGGTACAAGGCACATCCTGACG & $-1,+4$ & 1 \\
\hline & CAGTCGGTGGCTCAGCTACAGG-----TGGTACAAGGCACATCCTGACGA & -5 & 3 \\
\hline & CAGTCGGTGGCTCAGCTAC-----TGGTGGTACAAGGCACATCCTGACGA & -5 & 1 \\
\hline & CAGTCGGTGGCTCAGC--------TGGTGGTACAAGGCACATCCTGACGA & -8 & 2 \\
\hline & CAGTCGGTGGCTCAGCTACAGctcagctacagCTGGTGGTACAAGGCACATC & $-2,+11$ & 1 \\
\hline & CAGTCGGTGGCTCAGC---------------ggAAGGCACATCCTGACGAGT & $-17,+2$ & 1 \\
\hline & CAGTCGGTGGCTCAGCTACAGGC----------------------TGACGA & -21 & 1 \\
\hline & 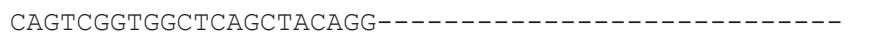 & -39 & 1 \\
\hline \multirow[t]{4}{*}{ GV (+LMB) } & CAGTCGGTGGCTCAGCTACAGGCC-GGTGGTACAAGGCACATCCTGACGA & -1 & 1 \\
\hline & CAGTCGGTGGCTCAGCTACAGG-----TGGTACAAGGCACATCCTGACGA & -5 & 1 \\
\hline & CAGTCGGTGGCTCAGCTACAGGCC---------AAGGCACATCCTGACGA & -9 & 2 \\
\hline & 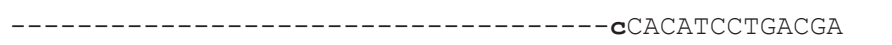 & $-170,+1$ & 2 \\
\hline
\end{tabular}

* The underlined nucleotides in the wild-type (WT) sequence shows a PAM domain. Small letters and hyphens indicate inserted and deleted nucleotides compared to WT, respectively. 
The absence of induced mutations in GV-arrested oocytes might be a result of the presence of a nuclear membrane barrier protecting the genome from Cas 9 attack, because the amount of Cas9 protein was lower in the nucleus than in the cytoplasm. Cas 9 is a large molecule with a molecular weight higher than $150 \mathrm{kDa}[6,14]$; the nuclear-import receptor, importin, is required for the transport of such macromolecular proteins into the nucleus [15]. The Cas 9 protein used in the present study contains the SV40 nuclear localization signal, which is recognized by importin, and the nuclear, especially nucleolar, localization of Cas 9 has been previously demonstrated in mouse pronuclear-stage embryos [6] and cultured human cells [2]. The low nuclear amount of Cas9 in GV oocytes that was observed in the present study implies a lower level of nuclear import activity in immature oocytes compared to embryos and somatic cells. Furthermore, the increase in the nuclear levels of Cas 9 caused by treatment with LMB, an inhibitor of exportin-1, indicates that Cas9 has a nuclear-export signal (NES) that exportin-1 recognizes as its target. In fact, we located two possible NESs in Cas9, LFGNLIALSL (aa 237-246) and LEDIVLTLTL (aa 616-625), using the NetNES v1.1 server (http://www.cbs.dtu.dk/services/NetNES/). However, further studies are needed to clarify whether these sites actually function as NESs. The mutation induction in GV oocytes by LMB treatment suggests the existence of a minimal Cas 9 concentration, below which CRISPR/Cas systems cannot function normally.

In most cases, one or two out of four alleles were mutated during the meiotic maturation of oocytes. Though the precise cause is unclear, the meiosis-specific extrusion of polar bodies can be ruled out as a possible explanation, because GV-arrested oocytes gave similar results. Because we evaluated mutation induction in oocyte genomes without separating polar bodies in the present study, it is unclear whether the mutated alleles were present in the oocytes or in the polar bodies. In any case, it should be noted that only one allele remained in the fertilized egg; the other three alleles were extruded in the first or the second polar body that were produced during meiosis. Therefore, the mutated allele may not be always inherited by the next generation. It would be interesting to analyze the dynamics of mutated alleles.

The results of the present study revealed that relatively short mutations were induced in maturing oocytes, whereas longer mutations were induced in the non-condensed chromosomes of LMB-treated GV oocytes. This may be attributed to difficulty of the resection factors in taking part in the NHEJ reaction to function in condensed chromosomes; therefore, hardly any large mutations are inserted [16]. However, as the mutation length depends on the target sequence, there is the possibility that this phenomenon was specific to the present target. Before we can draw a conclusion, further analyses including more target sequences and mutated alleles are clearly necessary.

The male and female genomes have not been distinguished from each other in genome modification studies thus far, with the exception of single study whose results suggested an asymmetric but sequential editing of male and female alleles [11]. The present study showed that a CRISPR/Cas system can be applied to maturing oocytes, which are cells having condensed chromosomes and contain genomes of female origin. Although the carry-over of Cas9 and gRNA to zygotes cannot be thoroughly dismissed, such an application could be helpful for functional analyses of sex-influenced genes, such as imprinted genes, through the preferential modification of female genomes. In addition, genome editing in oocytes could be useful in avoiding embryonic lethality by generating heterozygotic mutants after fertilization. More research into these applications and the optimization of mutagenesis in oocytes are expected to contribute to the further expansion of the already extensive use of CRISPR/ Cas systems.

\section{Acknowledgments}

This research was supported by a Grant-in-Aid for Scientific Research [25252056 to KN, 26712025 and $15 \mathrm{~K} 14884$ to WF and $16 \mathrm{~K} 15052$ to KS] from the Japan Society for the Promotion of Science.

\section{References}

1. Gaj T, Gersbach CA, Barbas CF 3rd. ZFN, TALEN, and CRISPR/Cas-based methods for genome engineering. Trends Biotechnol 2013; 31: 397-405. [Medline] [CrossRef]

2. Cong L, Ran FA, Cox D, Lin S, Barretto R, Habib N, Hsu PD, Wu X, Jiang W, Marraffini LA, Zhang F. Multiplex genome engineering using CRISPR/Cas systems. Science 2013; 339: 819-823. [Medline] [CrossRef]

3. Mali P, Yang L, Esvelt KM, Aach J, Guell M, DiCarlo JE, Norville JE, Church GM. RNA-guided human genome engineering via Cas9. Science 2013; 339: 823-826. [Medline] [CrossRef]

4. Shen B, Zhang J, Wu H, Wang J, Ma K, Li Z, Zhang X, Zhang P, Huang X. Generation of gene-modified mice via Cas9/RNA-mediated gene targeting. Cell Res 2013; 23: 720-723. [Medline] [CrossRef]

5. Wang H, Yang H, Shivalila CS, Dawlaty MM, Cheng AW, Zhang F, Jaenisch R. Onestep generation of mice carrying mutations in multiple genes by CRISPR/Cas-mediated genome engineering. Cell 2013; 153: 910-918. [Medline] [CrossRef]

6. Fujii W, Kawasaki K, Sugiura K, Naito K. Efficient generation of large-scale genomemodified mice using gRNA and CAS9 endonuclease. Nucleic Acids Res 2013; 41: e187. [Medline] [CrossRef]

7. Fujii W, Onuma A, Sugiura K, Naito K. Efficient generation of genome-modified mice via offset-nicking by CRISPR/Cas system. Biochem Biophys Res Commun 2014; 445: 791-794. [Medline] [CrossRef]

8. Fujii W, Onuma A, Sugiura K, Naito K. One-step generation of phenotype-expressing triple-knockout mice with heritable mutated alleles by the CRISPR/Cas9 system. J Reprod Dev 2014; 60: 324-327. [Medline] [CrossRef]

9. Hai T, Teng F, Guo R, Li W, Zhou Q. One-step generation of knockout pigs by zygote injection of CRISPR/Cas system. Cell Res 2014; 24: 372-375. [Medline] [CrossRef]

10. Han H, Ma Y, Wang T, Lian L, Tian X, Hu R, Deng S, Li K, Wang F, Li N, Liu G, Zhao Y, Lian Z. One-step generation of myostatin gene knockout sheep via the CRISPR/ Cas9 system. Front Agr Sci Eng 2014; 1: 2-5. [CrossRef]

11. Suzuki T, Asami M, Perry ACF. Asymmetric parental genome engineering by Cas 9 during mouse meiotic exit. Sci Rep 2014; 4: 7621. [Medline] [CrossRef]

12. Ohashi S, Naito K, Liu J, Sheng Y, Yamanouchi K, Tojo H. Expression of exogenous proteins in porcine maturing oocytes after mRNA injection: kinetic analysis and oocyte selection using EGFP mRNA. J Reprod Dev 2001; 47: 351-357. [CrossRef]

13. Whitworth KM, Lee K, Benne JA, Beaton BP, Spate LD, Murphy SL, Samuel MS, Mao J, OGorman C, Walters EM, Murphy CN, Driver J, Mileham A, McLaren D, Wells KD, Prather RS. Use of the CRISPR/Cas9 system to produce genetically engineered pigs from in vitro-derived oocytes and embryos. Biol Reprod 2014; 91: 78. [Medline] [CrossRef]

14. Jinek M, Chylinski K, Fonfara I, Hauer M, Doudna JA, Charpentier E. A programmable dual-RNA-guided DNA endonuclease in adaptive bacterial immunity. Science 2012; 337: 816-821. [Medline] [CrossRef]

15. Poon IKH, Jans DA. Regulation of nuclear transport: central role in development and transformation? Traffic 2005; 6: 173-186. [Medline] [CrossRef]

16. Chapman JR, Sossick AJ, Boulton SJ, Jackson SP. BRCA1-associated exclusion of 53BP1 from DNA damage sites underlies temporal control of DNA repair. J Cell Sci 2012; 125: 3529-3534. [Medline] [CrossRef] 